\title{
Sufficient Conditions and Applications for Carathéodory Functions
}

\begin{abstract}
Neng Xu
School of Mathematics and Statistics, Changshu Institute of Technology, Changshu, Jiangsu 215500, China

Correspondence should be addressed to Neng Xu; xun@cslg.edu.cn

Received 27 November 2012; Accepted 19 December 2012

Academic Editor: Jen-Chih Yao

Copyright (C) 2013 Neng Xu. This is an open access article distributed under the Creative Commons Attribution License, which permits unrestricted use, distribution, and reproduction in any medium, provided the original work is properly cited.

Let $P$ be the class of functions of the form $p(z)=1+\sum_{n=1}^{\infty} p_{n} z^{n}$ which are analytic in $U$. By using the method of differential subordinations, we give some sufficient conditions for Carathéodory function, that is, if $p(z) \in P$ satisfies $p(z) \neq 0(0<|z|<1)$ and $p(z)^{m}+\lambda\left(z p^{\prime}(z) /(p(z))^{\mu}\right) \prec h(z)$, where $h(z)=((1+a z) /(1+b z))^{m}+\lambda(a-b) z /(1+b z)^{2-\mu}(1+a z)^{\mu},-1 \leq b<a \leq 1, \lambda>0$, $m, \mu \in\{0,1,2\}, m+\mu \leq 2$, then $p(z) \prec(1+a z) /(1+b z)$. Some useful consequences of this result are also given.
\end{abstract}

\section{Introduction}

Let $A$ be the class of functions of the following form:

$$
f(z)=z+\sum_{n=2}^{\infty} a_{n} z^{n}
$$

which are analytic in $U=\{z:|z|<1\}$. We denote by $S$ the subclass of $A$ consisting of univalent functions and by $S^{*}$ and $K$ the usual subclasses of $S$ whose members are starlike (with respect to the origin) and close-to-convex, respectively. Finally, we denote by $R$ the family of functions $f \in A$ which satisfy the condition $\operatorname{Re} f^{\prime}(z)>0(z \in U)$. It is well known that $R \subset K$.

Let $P$ be the class of functions of the following form:

$$
p(z)=1+\sum_{n=1}^{\infty} p_{n} z^{n},
$$

which are analytic in $U$. If $p(z) \in P$ satisfies $\operatorname{Re} p(z)>$ $0(z \in U)$, then we say that $p(z)$ is the Carathéodory function. For Carathéodory functions, Nunokawa et al. [1] have shown some sufficient conditions applying the differential inequalities. In the present paper, using the method of differential subordinations, we derive certain conditions under which we have $p(z) \prec(1+a z) /(1+b z)$, where $-1 \leq b<a \leq 1$. Our results generalize or improve some results due to [1-4].

To prove our results, we need the following lemma due to Miller and Mocanu [5].
Lemma 1. Let $g(z)$ be analytic and univalent in $U$ and let $\theta(w)$ and $\varphi(w)$ be analytic in a domain D containing $g(U)$, with $\varphi(w) \neq 0$ when $w \in g(U)$. Set

$$
Q(z)=z g^{\prime}(z) \varphi(g(z)), \quad h(z)=\theta(g(z))+Q(z)
$$

and suppose that

(i) $Q(z)$ is starlike univalent in $U$, and

(ii) $\operatorname{Re}\left(z h^{\prime}(z) / Q(z)\right)=\operatorname{Re}\left\{\theta^{\prime}(g(z)) / \varphi(g(z))+z Q^{\prime}(z) /\right.$ $\mathrm{Q}(z)\}>0(z \in U)$.

If $p(z)$ is analytic in $U$, with $p(0)=g(0), p(U) \subset D$ and

$$
\begin{aligned}
\theta(p(z)) & +z p^{\prime}(z) \varphi(p(z)) \\
& \prec \theta(g(z))+z g^{\prime}(z) \varphi(g(z))=h(z),
\end{aligned}
$$

then $p(z) \prec g(z)$ and $g(z)$ is the best dominant of (4).

\section{Main Results}

Theorem 2. Let $-1 \leq b<a \leq 1, \lambda>0, m, \mu \in\{0,1,2\}$, and $m+\mu \leq 2$. If $p(z) \in P$ satisfies $p(z) \neq 0(0<|z|<1)$ and

$$
p(z)^{m}+\lambda \frac{z p^{\prime}(z)}{(p(z))^{\mu}} \prec h(z),
$$


where

$$
h(z)=\left(\frac{1+a z}{1+b z}\right)^{m}+\frac{\lambda(a-b) z}{(1+b z)^{2-\mu}(1+a z)^{\mu}},
$$

then $p(z) \prec(1+a z) /(1+b z)$ and $(1+a z) /(1+b z)$ is the best dominant of (5).

Proof. Let $-1 \leq b<a \leq 1, \lambda>0, m, \mu \in\{0,1,2\}$,

$$
D= \begin{cases}C & \mu=0, \\ C \backslash\{0\} & \mu=1,2,\end{cases}
$$

and choose

$$
g(z)=\frac{1+a z}{1+b z}, \quad \theta(w)=w^{m}, \quad \varphi(w)=\frac{\lambda}{w^{\mu}} .
$$

Then $g(z)$ is analytic and univalent in $U, g(0)=p(0)=1$, $p(U) \subset D, \theta(w)$ and $\varphi(w)$ satisfy the conditions of the lemma. The function

$$
Q(z)=z g^{\prime}(z) \varphi(g(z))=\frac{\lambda(a-b) z}{(1+b z)^{2-\mu}(1+a z)^{\mu}}
$$

is univalent and starlike in $U$ because

$$
\begin{aligned}
\operatorname{Re} \frac{z Q^{\prime}(z)}{Q(z)} & =1-(2-\mu) \operatorname{Re} \frac{b z}{1+b z}-\mu \operatorname{Re} \frac{a z}{1+a z} \\
& =-1+(2-\mu) \operatorname{Re} \frac{1}{1+b z}+\mu \operatorname{Re} \frac{1}{1+a z} \\
& >-1+(2-\mu) \frac{1}{1+|b|}+\mu \frac{1}{1+|a|} \geq 0 \quad(z \in U) .
\end{aligned}
$$

Further, we have

$$
\begin{aligned}
\theta(g(z))+Q(z) & =\left(\frac{1+a z}{1+b z}\right)^{m}+\frac{\lambda(a-b) z}{(1+b z)^{2-\mu}(1+a z)^{\mu}} \\
& =h(z), \\
\frac{z h^{\prime}(z)}{Q(z)} & =\frac{m}{\lambda}\left(\frac{1+a z}{1+b z}\right)^{m+\mu-1}+\frac{z Q^{\prime}(z)}{Q(z)} \quad(z \in U) .
\end{aligned}
$$

Since $m+\mu \leq 2$, from (11) it is easy to know that

$$
\operatorname{Re} \frac{z h^{\prime}(z)}{\mathrm{Q}(z)}>0 \quad(z \in U) \text {. }
$$

Hence the function $h(z)$ is close-to-convex and univalent in $U$. Now it follows from (5)-(12) that

$$
\begin{aligned}
\theta(p(z))+z p^{\prime}(z) \varphi(p(z)) \prec & \theta(g(z)) \\
& +z g^{\prime}(z) \varphi(g(z))=h(z) .
\end{aligned}
$$

Therefore, by virtue of the lemma, we conclude that $p(z) \prec$ $g(z)$ and $g(z)$ is the best dominant of (5). The proof of the theorem is complete.
Making use of the theorem, we can obtain a number of interesting results.

Corollary 3. Let $\beta>0, \alpha>0$. If $p(z) \in P$ satisfies

$$
\begin{aligned}
(p(z))^{2}+\frac{\beta}{\alpha} z p^{\prime}(z) & \prec \frac{\alpha+2(1+\beta) z+\alpha z^{2}}{\alpha(1-z)^{2}} \\
& =h_{1}(z) \quad(z \in U),
\end{aligned}
$$

then $\operatorname{Re} p(z)>0$.

Proof. Let $a=1, b=-1, \lambda=\beta / \alpha, \beta>0, \alpha>0, m=2$, and $\mu=0$ in the theorem, then we have

$$
\begin{array}{lc}
g(z)=\frac{1+z}{1-z}, & \theta(w)=w^{2}, \\
\varphi(w)=\frac{\beta}{\alpha}, & Q(z)=\frac{2 \beta z}{\alpha(1-z)^{2}}
\end{array}
$$

satisfy the conditions of the lemma. Note that

$$
\operatorname{Re} \frac{z h_{1}^{\prime}(z)}{\mathrm{Q}(z)}=\left(\frac{2 \alpha}{\beta}+1\right) \operatorname{Re} \frac{1+z}{1-z}>0 \quad(z \in U) .
$$

Hence, similar to the proof of the theorem, we conclude that $p(z) \prec g(z)$, that is, $\operatorname{Re} p(z)>0$.

Remark 4. Note that Corollary 3 was also proved by Nunokawa et al. [1] using another method.

Taking $a=1, b=-1, \lambda=\beta / \alpha, \alpha>0, \beta>0$, and $m=\mu=1$ in the theorem, we have the following.

Corollary 5. Let $\alpha>0, \beta>0, p(z) \in P$ satisfies $p(z) \neq 0(0<$ $|z|<1$ ), and

$$
\alpha p(z)+\beta \frac{z p^{\prime}(z)}{p(z)} \prec \alpha \frac{1+z}{1-z}+\frac{2 \beta z}{1-z^{2}}=h_{2}(z) \quad(z \in U),
$$

then $\operatorname{Re} p(z)>0$.

Remark 6. Note that the function

$$
w=h_{2}(z)=\alpha \frac{1+z}{1-z}+\frac{2 \beta z}{1-z^{2}} \quad(\alpha>0, \beta>0)
$$

maps $U$ onto the $w$-plane slit along the half-lines $\operatorname{Re} w=0$, $\operatorname{Im} w \geq \sqrt{\beta(2 \alpha+\beta)}$ and $\operatorname{Re} w=0, \operatorname{Im} w \leq-\sqrt{\beta(2 \alpha+\beta)}$. Hence Corollary 5 coincides with the result obtained by Nunokawa et al. [1, Theorem 2] using another method.

Letting $f(z) \in A, p(z)=f^{\prime}(z), \alpha=1$ in Corollary 5, we have the following.

Corollary 7. If $f(z) \in A, f^{\prime}(z) \neq 0(0<|z|<1)$, and

$$
f^{\prime}(z)+\beta \frac{z f^{\prime \prime}(z)}{f^{\prime}(z)} \neq i b \quad(z \in U),
$$

where $\beta>0$ is real and $|b| \geq \sqrt{\beta(2+\beta)}$, then $f(z) \in R$. 
Remark 8. Lewandowski et al. [3] proved if $f(z) \in A$, $f^{\prime}(z) \neq 0(0<|z|<1), \beta>0$, and

$$
\operatorname{Re}\left\{f^{\prime}(z)+\beta \frac{z f^{\prime \prime}(z)}{f^{\prime}(z)}\right\}>0 \quad(z \in U),
$$

then $f(z) \in R$. We see that Corollary 7 improves this result.

Corollary 9. Let $f(z) \in A$ with $f(z) f^{\prime}(z) \neq 0(0<|z|<1)$ If

$$
\frac{(z f(z))^{\prime \prime}}{f^{\prime}(z)}-2 \frac{z f^{\prime}(z)}{f(z)} \prec \frac{(1-\delta) z}{1+(1-\delta) z}=h_{3}(z)
$$

for some $\delta(0 \leq \delta<1)$, then $f(z) \in S$ and

$$
\left|\frac{z^{2} f^{\prime}(z)}{f^{2}(z)}-1\right|<1-\delta \quad(z \in U) .
$$

Proof. Letting $f(z) \in A, f(z) f^{\prime}(z) \neq 0(0<|z|<1)$, then $p(z)=z^{2} f^{\prime}(z) / f^{2}(z) \in P$,

$$
\frac{z p^{\prime}(z)}{p(z)}=\frac{(z f(z))^{\prime \prime}}{f^{\prime}(z)}-2 \frac{z f^{\prime}(z)}{f(z)} \text {. }
$$

Taking $m=0, \mu=\lambda=1$ and $a=1-\delta(0 \leq \delta<1)$, $b=0$ in the theorem, it follows from (5), (6), and (21) that $\left|\left(z^{2} f^{\prime}(z)\right) /\left(f^{2}(z)\right)-1\right|<1-\delta(z \in U)$, which implies that $f(z) \in S$ (see [6]).

Remark 10. Frassin and Darus [2] have shown that if $f(z) \in$ $A$ and

$$
\left|\frac{(z f(z))^{\prime \prime}}{f^{\prime}(z)}-2 \frac{z f^{\prime}(z)}{f(z)}\right|<\frac{1-\delta}{2-\delta} \quad(z \in U)
$$

for some $\delta(0 \leq \delta<1)$, then $\left|\left(z^{2} f^{\prime}(z) / f^{2}(z)\right)-1\right|<1-\delta(z \in$ $U)$.

For $0 \leq \delta<1$, the function

$$
h_{3}(z)=\frac{(1-\delta) z}{1-(1-\delta) z}
$$

is analytic and convex univalent in $U$. Since

$$
\begin{gathered}
h_{3}(U)=\left\{w:\left|w+\frac{(1-\delta)^{2}}{\delta(2-\delta)}\right|<\frac{1-\delta}{\delta(2-\delta)}\right\} \quad(0<\delta<1), \\
h_{3}(U)=\left\{w: \operatorname{Re} w<\frac{1}{2}\right\} \quad(\delta=0),
\end{gathered}
$$

the disk $|w|<(1-\delta) /(2-\delta)$ is properly contained in $h_{3}(U)$. Therefore, in view of (21), we see that Corollary 9 is better than the result given in [2].

Letting $f(z) \in A, p(z)=f^{\prime}(z) \in P, m=1, \mu=0$, $a=1-2 \alpha, 0 \leq \alpha<1$, and $b=-1$ in the theorem, we have the following.
Corollary 11. If $f(z) \in A, \lambda>0,0 \leq \alpha<1$, and

$$
\begin{aligned}
f^{\prime}(z)+\lambda z f^{\prime \prime}(z) \prec & \frac{1+(1-2 \alpha) z}{1-z} \\
& +\lambda \frac{2(1-\alpha) z}{(1-z)^{2}}=h_{4}(z) \quad(z \in U),
\end{aligned}
$$

then $\operatorname{Re} f^{\prime}(z)>\alpha$.

For the univalent function $h_{4}(z)$, we now find the image $h_{4}(U)$ of the unit disk $U$.

Let $h_{4}\left(e^{i \theta}\right)=u+i v$, where $u$ and $v$ are real. We have

$$
u=\alpha-\frac{(1-\alpha) \lambda}{1-\cos \theta}, \quad v=(1-\alpha) \frac{\sin \theta}{1-\cos \theta} .
$$

Elimination of $\theta$ yields

$$
v^{2}=\frac{2 \alpha(1-\alpha)-(1-\alpha)^{2} \lambda}{\lambda}-\frac{2(1-\alpha)}{\lambda} u
$$

Therefore, we conclude that

$$
\begin{gathered}
h_{4}(U)=\left\{w=u+i v: v^{2}>-\frac{2(1-\alpha)}{\lambda}\right. \\
\left.\times\left(u+\frac{(1-\alpha) \lambda}{2}-\alpha\right)\right\} .
\end{gathered}
$$

Remark 12. Nunokawa and Hoshino [4] have proved that if $f(z) \in A, \lambda>0$ and

$$
\operatorname{Re}\left\{f^{\prime}(z)+\lambda z f^{\prime \prime}(z)\right\}>-\frac{\lambda}{2}, \quad(z \in U)
$$

then $\operatorname{Re} f^{\prime}(z)>0$. For $\alpha=0, h_{4}(U)$ properly contains the half plane Re $w>-\lambda / 2$. Hence Corollary 11 with $\alpha=0$ is better than the result given in [4].

\section{Acknowledgments}

This work was partially supported by the National Natural Science Foundation of China (Grant no. 11171045). The author would like to thank the referees for their careful reading and for making some valuable comments which have essentially improved the presentation of this paper.

\section{References}

[1] M. Nunokawa, S. Owa, N. Takahashi, and H. Saitoh, "Sufficient conditions for Carathéodory functions," Indian Journal of Pure and Applied Mathematics, vol. 33, no. 9, pp. 1385-1390, 2002.

[2] B. A. Frasin and M. Darus, "On certain analytic univalent functions," International Journal of Mathematics and Mathematical Sciences, vol. 25, no. 5, pp. 305-310, 2001.

[3] Z. Lewandowski, S. Miller, and E. Złotkiewicz, "Generating functions for some classes of univalent functions," Proceedings of the American Mathematical Society, vol. 56, pp. 111-117, 1976.

[4] M. Nunokawa and S. Hoshino, "One criterion for multivalent functions," Proceedings of the Japan Academy. A, vol. 67, no. 2, pp. 35-37, 1991. 
[5] S. S. Miller and P. T. Mocanu, "On some classes of firstorder differential subordinations," The Michigan Mathematical Journal, vol. 32, no. 2, pp. 185-195, 1985.

[6] S. Ozaki and M. Nunokawa, "The Schwarzian derivative and univalent functions," Proceedings of the American Mathematical Society, vol. 33, pp. 392-394, 1972. 


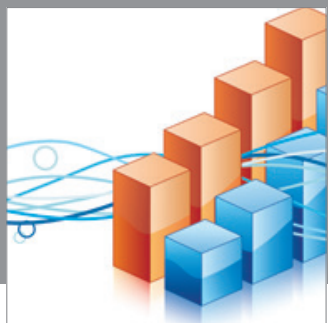

Advances in

Operations Research

mansans

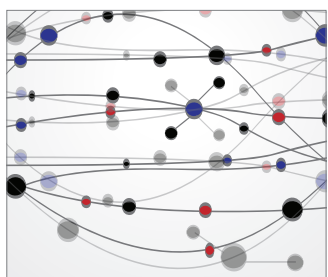

The Scientific World Journal
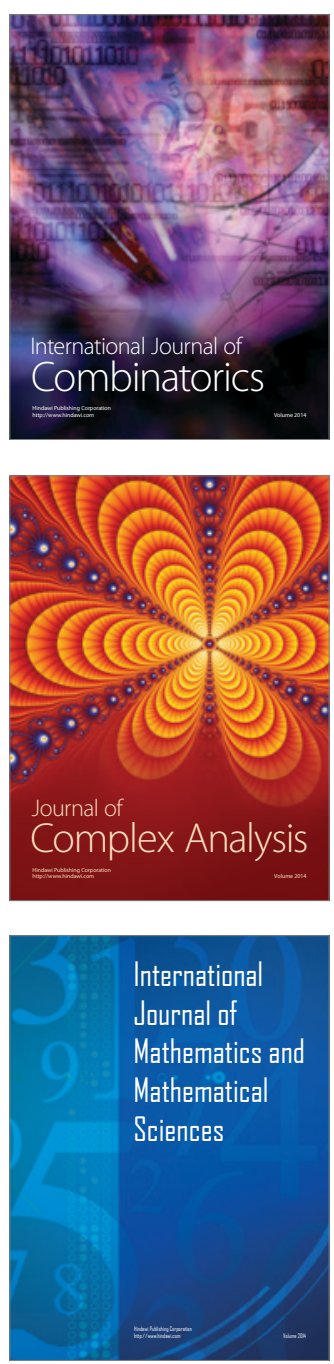
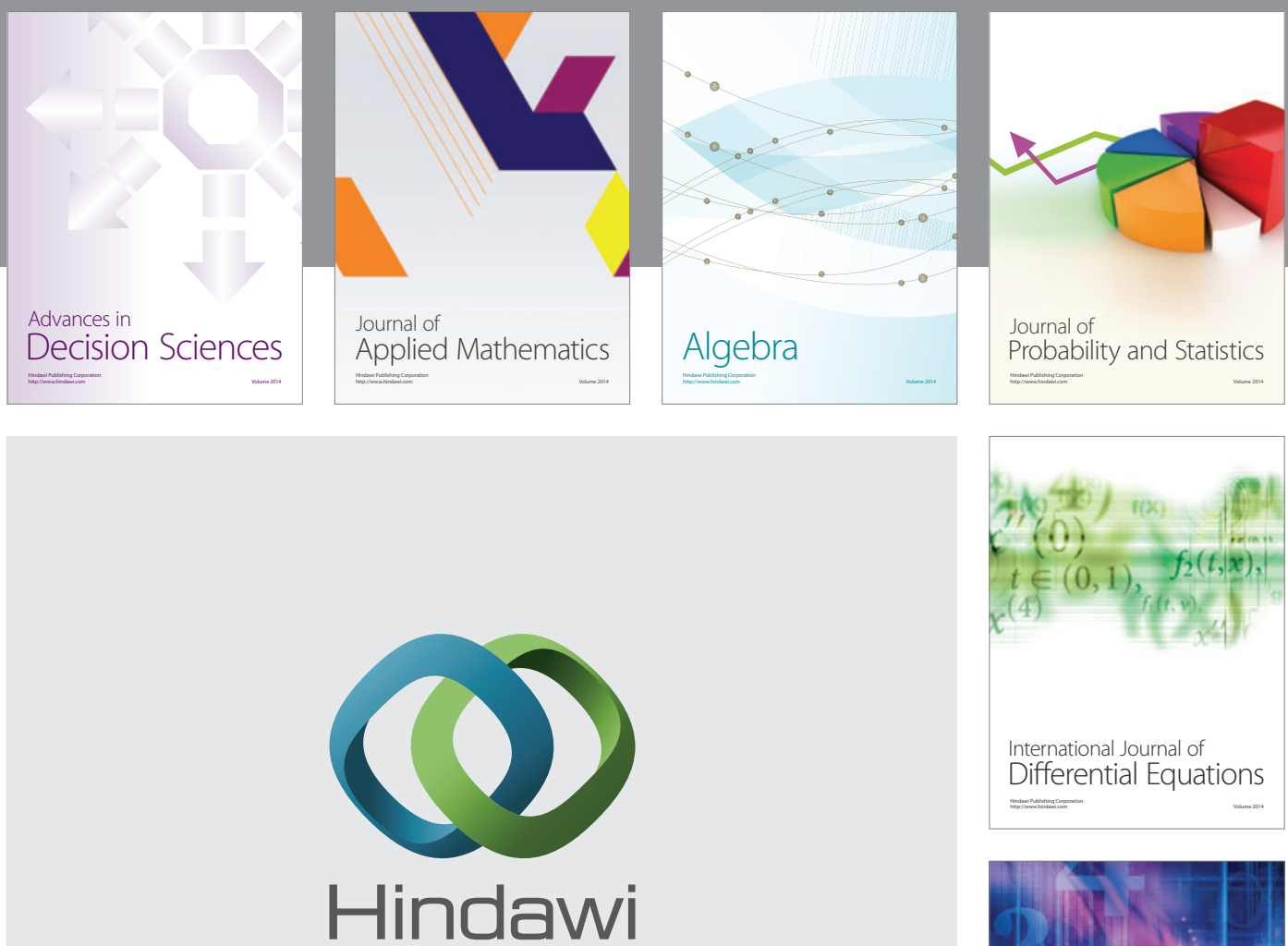

Submit your manuscripts at http://www.hindawi.com
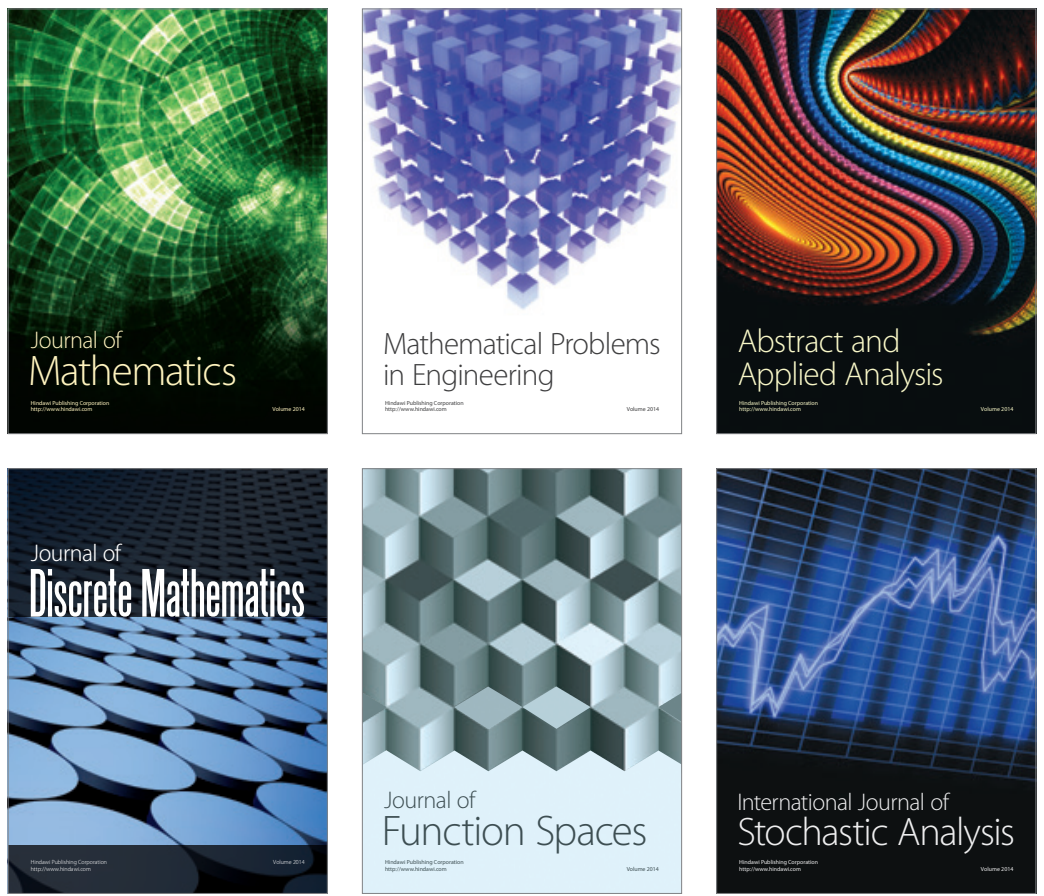

Journal of

Function Spaces

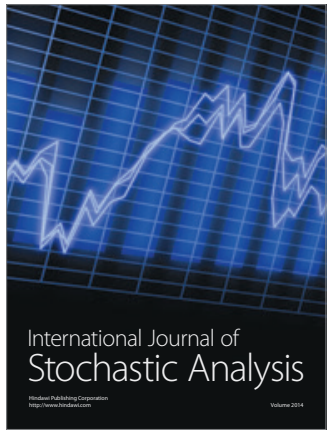

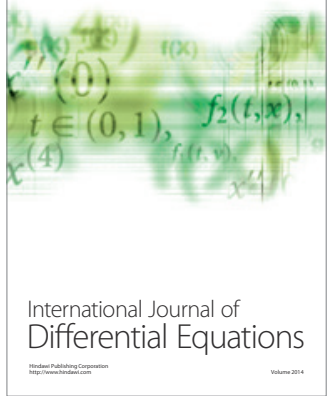
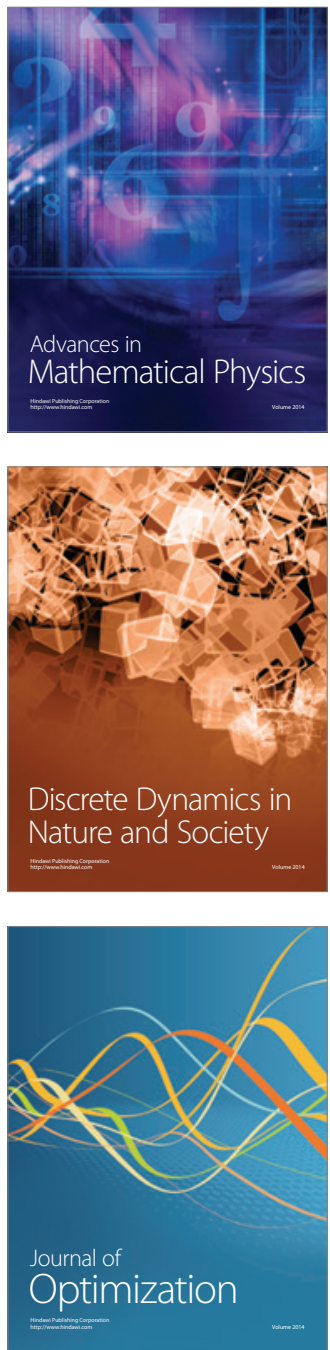